\title{
A new relict stem salamander from the Early Cretaceous of Yakutia, Siberian Russia
}

Pavel P. Skutschas, Veniamin V. Kolchanov, Alexander O. Averianov, Thomas Martin, Rico Schellhorn, Petr N. Kolosov, and Dmitry D. Vitenko

Acta Palaeontologica Polonica 63 (3), 2018: 519-525 doi:https://doi.org/10.4202/app.00498.2018

A new stem salamander, Kulgeriherpeton ultimum gen. et sp. nov., is described based on a nearly complete atlas (holotype) from the Lower Cretaceous (Berriasian-Barremian) Teete vertebrate locality in southwestern Yakutia (Eastern Siberia, Russia). The new taxon is diagnosed by the following unique combination of atlantal characters: the presence of a transversal ridge and a depression on the ventral surface of the posterior portion of the centrum; ossified portions of the intercotylar tubercle represented by dorsal and ventral lips; the absence of a deep depression on the ventral surface of the anterior portion of the centrum; the absence of pronounced ventrolateral ridges; the absence of spinal nerve foramina; the presence of a pitted texture on the ventral and lateral surfaces of the centrum and lateral surfaces neural arch pedicels; the presence of a short neural arch with its anterior border situated far behind the level of the anterior cotyles; moderately dorsoventrally compressed anterior cotyles; and the absence of a deep incisure on the distal-most end of the neural spine. The internal microanatomical organization of the atlas is characterized by the presence of a thick, moderately vascularized cortex and inner cancellous endochondral bone. The recognition of stem salamanders and other vertebrates with Jurassic affinities in the Early Cretaceous high-latitude (paleolatitude estimate $\mathrm{N} 63-70^{\circ}$ ) vertebrate assemblage of Teete suggests that: (i) the large territory of present day Siberia was a refugium for Jurassic relicts; (ii) there were no striking differences in the composition of high-latitude Yakutian and mid-latitude Western Siberian Early Cretaceous vertebrate assemblages; and (iii) there was a smooth transition from the Jurassic to Cretaceous biotas in North Asia.

Key words: Amphibia, Caudata, stem salamanders, Cretaceous, Russia, Siberia.

Pavel P. Skutschas [skutchas@mail.ru, p.skutschas@spbu.ru], Veniamin

V. Kolchanov [veniamin.kolchanov@mail.ru], and Dmitry D. Vitenko [mvitenko98@gmail.com

], Vertebrate Zoology Department, Saint Petersburg State University, Universitetskaya nab. 7/9, 199034 Saint Petersburg, Russia. Alexander O. Averianov [dzharakuduk@mail.ru], Zoological Institute of the Russian Academy of Sciences, Universitetskaya nab. 1, 199034 Saint Petersburg, Russia; Institute of Earth Sciences, Saint Petersburg State University, 16 Liniya VO 29, 199178 Saint Petersburg, Russia; Institute of Geology and Petroleum Technology, Kazan Federal 
University, Kremlevskaya str. 4/5, 420008 Kazan, Russia. Thomas Martin [tmartin@uni-bonn.de ] and Rico Schellhorn [rico.schellhorn@uni-bonn.de], Steinmann-Institut für Geologie, Mineralogie und Paläontologie, Universität Bonn, Nussallee 8, 53115 Bonn, Germany. Petr N. Kolosov [p_kolosov@diamond.ysn.ru], Institute of Diamond and Precious Metals Geology, Siberian Branch of the Russian Academy of Sciences, pr. Lenina 39, 677980 Yakutsk, Russia.

This is an open-access article distributed under the terms of the Creative Commons Attribution License (for details please see creativecommons.org), which permits unrestricted use, distribution, and reproduction in any medium, provided the original author and source are credited.

FoF 